\title{
Treino de dupla tarefa no tratamento fisioterapêutico da doença de Parkinson: uma
}

\section{revisão integrativa}

\author{
Dual-task training in the physical therapy treatment of Parkinson's disease: an integrative review \\ Entrenamiento de doble tarea en el tratamiento fisioterapéutico de la enfermedad de Parkinson:
} una revisión integradora

Recebido: 11/11/2021 | Revisado: 16/11/2021 | Aceito: 17/11/2021 | Publicado: 20/11/2021

\author{
Greyce Kelly Nascimento Sousa \\ ORCID: https://orcid.org/0000-0003-2353-4577 \\ Centro Universitário da Amazônia, Brasil \\ E-mail:7greyce@gmail.com \\ Isabele de Sousa Figueira \\ ORCID: https://orcid.org/0000-0001-9916-7326 \\ Centro Universitário da Amazônia, Brasil \\ E-mail: Isafigueira04@gmail.com \\ Paulo Henrique Ramos Pimentel \\ ORCID: https://orcid.org/0000-0002-9561-5715 \\ Centro Universitário da Amazônia, Brasil \\ E-mail: pauloramosfisio@hotmail.com
}

\begin{abstract}
Resumo
Objetivos: Investigar os benefícios da utilização do treino de dupla tarefa no tratamento da Doença de Parkinson. Métodos: Trata-se de uma revisão integrativa, onde foram utilizados 03 trabalhos para compor este trabalho. Base dados utilizada Google acadêmico com trabalhos publicados nos últimos 10 anos, sendo pesquisados trabalhos nos idiomas ingleses e portugueses. Resultados: O estudo permitiu a analise acerca dos portadores de DP, quanto ao desempenho das atividades motoras de dupla tarefa, o grupo portador da doença de Parkinson apresentou apuro no desenvolvimento das tarefas levando em consideração o tempo e a dificuldade do exercício proposto, já o grupo de idosos que não possuem DP o tempo e a dificuldade da atividade proposta foram considerados menor. Conclusão: A partir da conclusão deste estudo observou-se a importância das atividades de duplas tarefas para os portadores da Doença de Parkinson, por serem simples atividades estas são eficazes nas atividades desenvolvidas, com o intuito de estimular fala, movimentos, rigidez muscular, treino de marcha e entre outros.
\end{abstract}

Palavras-chave: Doença de Parkinson; Atividade motora; Análise da marcha.

\begin{abstract}
.
Objectives:To investigate the benefits of dual task training in the treatment of Parkinson's disease. Methods: This is an integrative review, where 03 papers were used to compose this work. The database used was Google Scholar, with papers published in the last 10 years, and researched papers in English and Portuguese. Results: the study allowed the analysis of the elderly with PD, as to the performance of motor activities of dual task, the group with Parkinson's disease showed improvement in the development of tasks taking into account the time and difficulty of the exercise proposed, while the group of elderly who do not have PD the time and difficulty of the activity proposed was considered lower. Conclusion: From the conclusion of this study we observed the importance of dual task activities for people with Parkinson's disease, because they are simple activities that are effective in the developed activities, with the aim of stimulating speech, movement, muscle stiffness, gait training and others.
\end{abstract}

Keywords: Parkinson's disease; Motor activity; Gait analysis.

\section{Resumen}

Objetivos: Investigar los beneficios del entrenamiento de tareas duales en el tratamiento de la enfermedad de Parkinson. Métodos: Se trata de una revisión integradora, en la que se utilizaron 03 artículos para componer este trabajo. La base de datos utilizada es la de Google academic con artículos publicados en los últimos 10 años, siendo buscados artículos en inglés y portugués. Resultados: el estudio permitió el análisis sobre los pacientes con EP, en cuanto a la realización de actividades motrices de doble tarea, el grupo con enfermedad de Parkinson mostró una mejora en el desarrollo de las tareas teniendo en cuenta el tiempo y la dificultad del ejercicio propuesto, mientras que el grupo de mayores que no tienen EP el tiempo y la dificultad de la actividad propuesta se consideró menor. Conclusión: A partir de la conclusión de este estudio se observó la importancia de las actividades de tareas dobles para los portadores de la enfermedad de Parkinson, por ser actividades simples estas son efectivas en las actividades 
desarrolladas, con la intención de estimular el habla, los movimientos, la rigidez muscular, el entrenamiento de la marcha y entre otros.

Palabras clave: Enfermedad de Parkinson; Actividad motora; Análisis de la marcha.

\section{Introdução}

Os distúrbios de equilíbrio em indivíduos com Doença de Parkinson (DP) podem se manifestar em diferentes estágios da doença, aumentando sua incapacidade física. Isso pode ser justificado pelo comprometimento da habilidade do sistema nervoso central em processar as aferências vestibulares, visuais e proprioceptivas, as quais são interpretadas pelo acometido para gerar as respostas musculares adequadas responsáveis pela manutenção do equilíbrio corporal (Terra, et al. 2016). Apresenta sinais de rigidez, acinesia, bradicinesia, tremor e instabilidade postural. De início pérfido, lento e gradativo. Figurase de etiologia idiopática, porém, acredita-se que pode surgir de agentes ambientais e genéticos, causando um desenvolvimento neurodegenerativo da Dopamina. Silva et al. (2017) [3], aponta que o mau desempenho de equilíbrio gera prejuízos funcionais para o idoso causando quedas e maiores níveis de morbidade e mortalidade nessa população.

A dupla tarefa (DT), também conhecida como tarefa simultânea ou associada, é aprendida durante todo o curso de vida de um indivíduo. É um pré-requisito para o desempenho funcional em várias atividades da vida diária (Hallal C. Z. et al. 2013). A realização de dupla tarefa envolve a atividade de uma principal - a tarefa simples, o principal foco de atenção e uma tarefa secundária desenvolvida simultânea. Com o avançar da idade a execução da DT se torna mais deficitária ocasionando o maior risco de quedas em idosos, pois a maior parte das atividades da vida diária envolve a mesma. E quando existe uma limitação cognitiva e/ou motora, tem-se a necessidade de uma atenção maior, podendo assim levar a um comprometimento do funcionamento de ambas as tarefas (O'she S, et al. 2002; Silva, et al. 2017).

Alguns estudos apontam que em decorrência da perda de neurônios da parte compacta da substância negra, ocasiona alterações posturais e distúrbios de equilíbrio e marcha, tendo sua severidade e prevalência aumentadas com a progressão da doença. Relatam também, que uma das condições que corroboram para o risco de queda é um mau desempenho na realização de atividades de dupla tarefa. Com o prolongar da idade a execução da dupla tarefa tenha uma diminuição, de modo a ocasionar a redução de movimentos em um espaço de tempo e em casos mais avançados levando a não realização destes.

Levando-se em consideração que para se ter uma boa qualidade de vida uma pessoa tenha condições de realizar mais de uma tarefa ao mesmo tempo, desde ao falar enquanto caminha ou ao realizar alguma atividade da vida diária, da qual se necessita para ter independência dentro de casa. Estudos relatam que o avançar da idade torna os idosos restritos a realização das tarefas diárias, uma vez que a maior parte das atividades da vida diária envolve dupla tarefa. E quando existe uma limitação cognitiva e/ou motora, tem-se a necessidade de uma atenção maior, podendo assim levar a um comprometimento do funcionamento de ambas as tarefas. Pessoas com Doença de Parkinson tendem a não realizar movimentos sequenciais automáticos, constituindo a marcha como sendo a mais incapacitante nesse grupo de indivíduos.

Sousa et al. (2014) [2], caracteriza a marcha na Doença de Parkinson por algumas alterações variáveis espaçostemporais e angulares, como diminuição na velocidade, no comprimento da passada e no balanceio dos membros superiores, aumento compensatório na cadência e na variabilidade entre as passadas, redução na amplitude de extensão do quadril na fase de apoio médio, na flexão de joelho durante o balanço e na flexão plantar durante a fase de impulsão. Um indivíduo capaz de realizar alguma tarefa secundaria enquanto caminha obtém grandes vantagens, pois assim impossibilita incidentes relacionados a marcha.

Pacientes com Doença de Parkinson devem ser submetidos a terapias de reabilitação para evitar o máximo de agravamento. Evidencias sugerem que a interferência de métodos cognitivos ou motores-cognitivos associado tem efeito benéfico para o funcionamento físico, controle postural e marcha. 
Esta revisão integrativa tem como objetivo investigar os benefícios da utilização do treino de dupla tarefa no tratamento da Doença de Parkinson.

\section{Metodologia}

Concerne de uma revisão integrativa, que segundo Sousa et al, emerge como uma metodologia que proporciona a síntese de conhecimento e a incorporação da aplicabilidade de resultados de estudos significativos na prática. Foram inclusos estudos de casos e ensaios clínicos que tenham sido publicados em português e/ou inglês dos últimos 10 anos (2011-2021), que correspondam ao objetivo desse projeto.

A aquisição de conteúdo para este estudo foi realizada através de busca na plataforma: SciELO, PubMed, PeDro, com os descritores português e inglês: Doença de Parkinson (Parkinson`s desease) e atividade motora (Motor Activity) e Análise da Marcha (Gait Analysis). Onde foram encontrados 3.970 resultados para busca, no qual três (3) foram selecionados para compor este trabalho após uma análise por seguirem os critérios de inclusão (estudos publicados entre 2011 e 2021, estudos que não fizessem uso de animais, e que correspondessem ao tema abordado neste trabalho).

Em seguida fora feita a leitura exploratória do material adquirido, para assim serem definidos os estudos que seriam utilizados para compor este projeto, seguido da organização dos dados obtidos (conceitos, grupo estudado, amostragens, resultados e referencias). Finalizando assim com uma análise e comparação dos resultados obtidos pelos pesquisadores.

O Quadro 1, a seguir, apresenta: resultados da pesquisa: 
Quadro 1. Resultados da pesquisa.

\begin{tabular}{|c|c|c|c|c|c|}
\hline $\mathbf{N}$ & Título & Autores/Ano & Amostras/Idade & Intervenção & Resultados \\
\hline 1 & $\begin{array}{lr}\text { Impacto da doença de } \\
\text { Parkinson } & \text { na } \\
\text { performance } & \text { do } \\
\text { equilíbrio } & \text { em } \\
\text { diferentes } & \text { demandas } \\
\text { atencionais. } & \end{array}$ & $\begin{array}{l}\text { TERRA, Marcelle } \\
\text { Brandão et al. } \\
(2016) \text {. }\end{array}$ & $\begin{array}{l}19 \text { participantes com } \\
\text { idade acima de } 50 \\
\text { anos. }\end{array}$ & $\begin{array}{l}\text { Os pacientes foram } \\
\text { avaliados em uma } \\
\text { plataforma de força } \\
\text { Biomec400. Os } \\
\text { parâmetros analisados } \\
\text { foram a área do centro } \\
\text { de pressão dos pés } \\
\text { (COP) e a amplitude e } \\
\text { velocidade do COP } \\
\text { nas direções } \\
\text { anteroposterior (AP) e } \\
\text { a mediolateral (ML). }\end{array}$ & $\begin{array}{l}\text { Indivíduos com DP, } \\
\text { quando submetidos a } \\
\text { desafios cognitivos, } \\
\text { comportam-se de } \\
\text { forma semelhante à } \\
\text { retirada do recurso } \\
\text { visual no que diz } \\
\text { respeito às alterações } \\
\text { de equilíbrio. }\end{array}$ \\
\hline 2 & $\begin{array}{l}\text { Desempenho em } \\
\text { atividades de simples } \\
\text { e dupla tarefa de } \\
\text { idosos } \\
\text { institucionalizados que } \\
\text { realizam e não } \\
\text { realizam fisioterapia. }\end{array}$ & $\begin{array}{l}\text { SILVA, Rubia } \\
\text { Jaqueline } \\
\text { Magueroski da, } \\
\text { DIAS, Sara Maria } \\
\text { Soffiatti, PIAZA, } \\
\text { Lisiane. } \\
\text { (2017). }\end{array}$ & $\begin{array}{l}60 \text { participantes, igual } \\
\text { ou acima de } 60 \text { anos. }\end{array}$ & $\begin{array}{l}\text { Foram realizadas as } \\
\text { seguintes atividades: } \\
\text { ficar em apoio } \\
\text { unipodal, caminhar ao } \\
\text { longo de um corredor } \\
\text { por } 30 \text { segundos, subir } \\
\text { e descer de um step } \\
\text { por } 30 \text { segundos, } \\
\text { sentar e levantar de } \\
\text { uma cadeira por } 30 \\
\text { segundos (tarefa } \\
\text { simples). Depois, os } \\
\text { idosos realizaram as } \\
\text { mesmas atividades } \\
\text { segurando um copo de } \\
\text { plástico com água } \\
\text { (dupla tarefa motora), } \\
\text { dizendo os dias da } \\
\text { semana ao contrário } \\
\text { (dupla tarefa } \\
\text { cognitiva) e segurando } \\
\text { um copo de água e } \\
\text { dizendo os dias da } \\
\text { semana ao contrário } \\
\text { (dupla tarefa motora e } \\
\text { cognitiva). }\end{array}$ & $\begin{array}{l}\text { Foi observada uma } \\
\text { redução no } \\
\text { desempenho funcional } \\
\text { de idosos } \\
\text { institucionalizados que } \\
\text { realizavam e não } \\
\text { realizavam fisioterapia } \\
\text { ao adicionar uma } \\
\text { dupla tarefa nas } \\
\text { atividades de caminhar } \\
\text { e sentar e levantar de } \\
\text { uma cadeira; } \\
\text { demonstrando que ao } \\
\text { adicionar a dupla } \\
\text { tarefa ocorre impacto } \\
\text { negativo, dificultando } \\
\text { a execução das } \\
\text { atividades. }\end{array}$ \\
\hline 3 & $\begin{array}{l}\text { Duplo desempenho de } \\
\text { tarefas: uma } \\
\text { comparação entre } \\
\text { indivíduos saudáveis e } \\
\text { aqueles com doenças } \\
\text { de Parkinson. }\end{array}$ & $\begin{array}{l}\text { FLORIANO, } \\
\text { Eduardo } \\
\text { Nascimento, et al. } \\
(2015)\end{array}$ & $\begin{array}{lr}21 & \text { indivíduos } \\
\text { saudáveis e } \quad 21 \\
\text { indivíduos com Doença } \\
\text { de Parkinson com } \\
\text { idade média de } 50 \text { a } 75 \\
\text { anos }\end{array}$ & $\begin{array}{l}\text { Os participantes foram } \\
\text { submetidos a cinco } \\
\text { tarefas simples. } \\
\text { (motoras) e a cada } \\
\text { uma delas foi } \\
\text { associado uma tarefa } \\
\text { cognitiva, } \\
\text { caracterizando uma } \\
\text { DT. } \\
\text { Para avaliação do } \\
\text { equilíbrio e da marcha } \\
\text { foram utilizados os } \\
\text { seguintes } \\
\text { instrumentos: Escala } \\
\text { de Equilíbrio de Berg, } \\
\text { Escala de Tinetti, e } \\
\text { Índice de Marcha } \\
\text { Dinâmica. }\end{array}$ & $\begin{array}{l}\text { Este estudo permitiu } \\
\text { verificar que o grupo } \\
\text { de indivíduos com a } \\
\text { doença de Parkinson } \\
\text { teve um desempenho } \\
\text { pior que indivíduos } \\
\text { saudáveis em tarefas } \\
\text { simultâneas e } \\
\text { necessitou de mais } \\
\text { tempo para realizar as } \\
\text { mesmas tarefas. }\end{array}$ \\
\hline
\end{tabular}

Fonte: Autores.

\section{Discussão}

A Doença de Parkinson tem acometido grande parte dos idosos, esta mazela afeta principalmente os movimentos comprometendo as atividades básicas do cotidiano, com movimentos cada vez mais lentos, alterações na comunicação, falta de 
equilíbrio, entre outros. Neste estudo foram utilizados três (3) artigos com diversos idosos de faixa etárias diferentes, todos passaram por diversas atividades motoras que consistiam na realização de exercícios de dupla tarefa, com o intuito de analisar e comparar o desempenho de cada indivíduo que participava de cada pesquisa.

O estudo transversal de Terra et al. (2016). [1], objetivou avaliar o equilíbrio na posição tandem de olhos abertos (OA), tandem com os olhos fechados (OF) e tandem na condição de dupla tarefa (DT). Sua amostra constou de 19 participantes acima de 50 anos diagnosticados com doença de Parkinson idiopática segundo os critérios do banco de cérebro de Londres, no estágio leve a moderado de acordo com a escala de Hoehn \& Yahr (HY) modificado, independentes para deambular e não inseridos em outros programas terapêuticos além do medicamentoso.

Todos os procedimentos de avaliação foram realizados no período da medicação, com a utilização dos seguintes instrumentos: Escala de HY modificado: avalia o estadiamento da doença e a incapacidade dos indivíduos com Doença de Parkinson. Para o estudo foram selecionados os pacientes classificados entre os estágios 1,5 e 3 (incapacidade leve a moderada); Unified Parkinson’s Disease Rating Scale (UPDRS): Avalia a progressão da doença de acordo com suas características clinicas, composta por 42 itens, divididos em 04 domínios. A pontuação em cada item varia de 0 a 4 sendo que quanto maior a pontuação, maior o comprometimento da doença. Foram utilizados os domínios de atividades de vida diária e exame motor; Miniexame do estado mental (MEEM):Avalia as funções cognitivas, compostas por questões agrupadas em sete categorias, cada uma delas com o objetivo de avaliar funções cognitivas especificas como orientação para tempo e espaço, registro e lembrança de palavras, linguagem, atenção, cálculo e capacidade construtiva visual. $\mathrm{O}$ escore pode variar de 0 a 30 pontos, e o ponto de corte 24 tem de boa a excelente sensibilidade e especificidade para o diagnóstico da demência.

As atividades foram feitas por 30 segundos cada, com intervalo de repouso, e foi obtida a média dos valores das duas tentativas para as análises. Os dados foram analisados de acordo com a distribuição da normalidade e representado segundo a média e o desvio padrão, utilizando Analysis oef Variance (Anova). Foi utilizado o pós-teste de Tukey para obter valores de diferença mínima significante.

Os autores concluíram que indivíduos com Doença de Parkinson, quando submetido a desafios cognitivos, comportam-se de forma semelhante à retirada do recurso visual no que diz respeito às alterações de equilíbrio.

Já Silva et al. (2017). [3], realizou um estudo de caráter transversal, descritivo e comparativo. Objetivando comparar o desempenho em atividades de tarefa simples e de dupla tarefa de idosos institucionalizados que realizam e não realizam fisioterapia. Participaram do estudo 60 indivíduos, de ambos os sexos, sendo divididos em dois grupos: 30 idosos institucionalizados que realizavam fisioterapia (GF) e 30 idosos institucionalizados que não realizavam fisioterapia (GNF). Todos foram escolhidos de forma não probabilística intencional. Idade igual ou acima de 60 anos, capacidade de locomoção independente, sem diagnóstico estabelecido de disfunção cognitiva e residir em uma instituição de longa permanência da Grande Florianópolis. Idosos que realizavam fisioterapia pelo menos duas vezes na semana e idosos que não realizavam fisioterapia

$\mathrm{Na}$ execução do estudo foram utilizados os seguintes materiais: cronometro, fita métrica, step, cadeira e um copo plástico com água. Fora também aplicada um questionário que possibilita calcular o tempo semanal despedindo em atividades físicas de intensidade moderada e vigorosa, em inúmeras situações da vida (IPAQ). Em seguida, os idosos foram submetidos à avaliação do seu desempenho na realização de atividades de tarefa simples (TS) e dupla tarefa (DT).

Para análise estatística foi utilizado o Statistical Package for the Social Sciences (SPSS v. 20.0). A estatística descritiva foi utilizada para caracterização dois sujeitos e o teste Shapiro-Wilk foi utilizado para verificar a normalidade de estudo. Teste independente para comparar idade e o tempo de institucionalização entre os grupos. Para verificar o efeito da dupla tarefa e da fisioterapia sobre as atividades de apoio unipodal, caminhar, subir e descer de um step e sentar e levantar de uma cadeira, foi utilizada a Anova fatorial 2x4 com correção de Bonferroni. 
Com este estudo foi observada uma redução no desempenho funcional de idosos institucionalizados que realizavam e não realizavam fisioterapia ao adicionar uma dupla tarefa nas atividades de caminhar e sentar e levantar de uma cadeira; demonstrando que ao adicionar a dupla tarefa ocorre impacto negativo dificultando a execução das atividades.

No estudo transversal de Floriano et al. (2015). [4]o objetivo foi comparar o equilíbrio, a marcha e a tarefa simples e dupla de indivíduos saudáveis e indivíduos com Doença. Foram utilizados os seguintes instrumentos para avaliar o equilíbrio e a marcha: 1) Berg Balance Scale e o teste de Tinetti, que destinam a avaliar o equilíbrio em diferente atividade da vida diária. A pontuação máxima de Berg é 56 e a pontuação máxima de Tinetti é 28; 2) Índice Dinâmico de Andamento foi usado para avaliar os indivíduos em diferentes situações tais como andar em diferente velocidade, caminhar olhando para os lados subir e descer atravessar e caminhar em torno de obstáculo, subir e descer escada. A pontuação máxima dessa escala é 24; 3) Para avaliar o desempenho em contexto simples de dupla tarefa foi solicitado no estágio "on" de medicina, que realizassem cinco tarefa simples (motoras) e depois cada uma destas tarefas foi associada a uma cognitiva.

Este estudo permitiu verificar que o grupo de indivíduos com a Doença de Parkinson teve um desempenho pior que os indivíduos saudáveis em tarefas simultâneas e necessitou de mais tempo para realizar as mesmas tarefas.

Os estudos apresentados nesta revisão usaram de técnicas e métodos variados para avaliação e teste de pessoas com Doença de Parkinson com relação ao treino de dupla tarefa. Apresentando níveis de dificuldades variadas de acordo com a finalidade de cada pesquisa, no estudo de Terra fora testado o equilíbrio em relação às dificuldades visuais e desafios cognitivos, enquanto no de Silva os idosos foram testados na realização de tarefas simples e dupla tarefa mostrando assim grande dificuldade na realização destas. Já para Floriano, a comparação entre indivíduos saudáveis e com Doença de Parkinson apenas ressaltou o grau de dificuldade em desempenhar a dupla tarefa por pacientes diagnosticados com DP. Chegando a concluir que quando realizada a dupla tarefa os indivíduos acometidos por esta patologia tendem a ter um grau mais elevado de dificuldade e sofrem impacto negativo em relação às atividades da vida diária, e a marcha é a mais acometida neste grupo estudado.

\section{Conclusão}

A partir da conclusão deste estudo observou-se a importância das atividades de duplas tarefas para os portadores da Doença de Parkinson, por serem simples atividades estas são eficazes nas atividades desenvolvidas, com o intuito de estimular fala, movimentos, rigidez muscular, treino de marcha e entre outros, a grande maioria dos portadores são idosos que necessitam de cuidados, devido a essa escassez de novos estudos é necessário ainda mais ensaios clínicos, investimentos em pesquisas para verificar protocolos de intervenção e os efeitos da intervenção da atividade de dupla tarefa em pessoas com doença de Parkinson. Além de novos estudos referente a esta doenças.

\section{Agradecimentos}

Agradecemos primeiramente a Deus por até aqui ter nos sustentado com força, sabedoria durante todo este trabalho. Gratidão pelo apoio incondicional recebido de nossas famílias ao longo de nossas vidas, apoio financeiro e principalmente emocional e ao nosso querido amigo Elieldo Vitor Figueira pelo apoio durante o desenvolvimento desta empreitada.

Agradecimento especial ao nosso orientador Dr. Paulo Henrique Ramos Pimentel pelo empenho e encorajamento para conosco durante a caminhada para aqui chegarmos. Queremos agradecer a todos aos nossos professores que foram essenciais ao longo desta jornada. 


\section{Referências}

Almeida, I. A. et al. (2015). Fisioterapia no treinamento de dupla tarefa no equilíbrio de indivíduos com doença de Parkinson. Saúde. 41(2), 71-80.

Araújo, F. R. et al. (2020). Interferência dupla tarefa no desempenho da marcha em indivíduos com doença de Parkinson. Revista Pesquisa em Fisioterapia, 10 (2): $248-57$

Barbosa, J. M. M. et al. (2008). Efeito da realização simultânea de tarefas cognitivas e motoras no desempenho funcional de idosos da comunidade. Fisioterapia e Pesquisa, 15(4), 374-9.

Bueno, M. E. et al. (2014). Efetividade da fisioterapia com treinamento de dupla tarefa no sistema motor e cognitivo em indivíduos com doença de Parkinson. Revista Saúde e Pesquisa, 7 (2): 241-49.

Candido, D. P. et al. (2012). Analise dos efeitos da dupla tarefa na marcha de pacientes com doença de Parkinson: relato de três casos. Rev. Neurocienc. 20(2): 240-245. https://doi.org/10.34024/rnc.2012.v20.8289

Carlos, I. S., Gonçalves, G. B., Pereira, J. S. (2015). Influencia do treino de dupla tarefa no desempenho motor e funcional de parkinsonianos. HU Revista, 41(1 e 2), 71-77.

Fatori, C. O., et al. (2015). Dupla tarefa e mobilidade funcional de idosos ativos. Rev. Bras. Geriatr. Gerontol., 18(1): 29-37. https://dx.doi.org/10.1590/18099823.2015.13180

Felippe, L. A. et al. (2014). Funções executivas, atividades da vida diária e habilidade motora de idosos com doenças neurodegenerativas. $J$ Bras Psiquiatr. 63(1): 39-47. https://dx.doi.org/10.1590/0047-2085000000006

Floriano, E. N. et al. (2015). Duplo desempenho de tarefas: uma comparação entre indivíduos idosos saudáveis e aqueles com doença de parkinson. Fisioter Mov. 28(2):251-58.

Maciel, M. A. et al. (2014). Impacto da dupla tarefa na marcha de pacientes com doença de Parkinson, acidente vascular cerebral e ataxia: uma análise comparativa. Psycology/Psicologia e Crítica, 27 (2), 351-57.

Marinho, M. S. et al. (2014). Dupla-tarefa na doença de Parkinson: uma revisão sistemática de ensaios clínicos aleatorizados. Rev. Bras. Geriatr. Gerontol. https://doi.org/10.1590/s1809-9823201400018

Mendel, T. et al. (2015). Dupla tarefa como estratégia terapêutica em fisioterapia neurofuncional: uma revisão de literatura. Acta. Fisiatr. 22 (4): $206-11$.

Pinto, E. S. M. et al. (2015). Influência da dupla tarefa na esteira ergométrica sobre a doença de Parkinson. Ciência em Movimento. Reabilitação e Saúde. 55(17).

Silva, R. J. M. et al. (2017). Desempenho em atividades de simples e dupla tarefas de idosos institucionalizados que realizam e não realizam fisioterapia. Fisioter. Pesqui. 24(2): 149-156. 10.1590/1809-2950/16610424022017

Silva, R. J. M. et al. (2017). Institucionalizados que realizam fisioterapia. Fisioter Pesqui. 24(2):149-56.

Silva, R. N. et al. (2021). Intervenção de dupla tarefa com base no teste de trilha: efeitos sobre a doença de Parkinson. Jounal of Bodywork and Movement Therapies. 27, 628-33.

Sousa, A. V. C. et al. (2014). Influência do treino em esteira na marcha em dupla tarefa em indivíduos com doença de parkinson: um estudo de caso. Fisioter Pesqui. 21(3):291-6.

Teixeira, N. B., Alouder, S. R. (2007). O desempenho da dupla tarefa na doença de Parkinson. Rev. Bras. Fisioter., 11(2), 127-132,

Terra, M. I. B. et al. (2016) Impacto da doença de parkinson na performance do equilíbrio em diferentes demandas atencionais. Fisioter Pesqui. 23 (4):410-5.

Tomo, C. K. et al. (2014). Efeitos do treino funcional de membro superior em condição de dupla tarefa na doença de Parkinson. Rev. Neurocienc. 22(3): 344350. 10.4181/RNC.2014.22.03.966.7p 